\title{
Low Cost IP Private Branch Exchange (PBX)
}

\author{
Prof.S.D.Giripunje \\ Department of Electronics Engineering, \\ G.H.Raisoni College of Engineering, \\ Digdoh Hills, Hingna,Ngapur INDIA
}

\author{
Sandeep Sonaskar \\ Research Scholar, Department of Electronics \& \\ Telecommunication Engineering, \\ G.H.Raisoni College of Engineering, \\ Digdoh Hills, Hingna, Ngapur INDIA
}

\begin{abstract}
The drawback of EAPBX system is that it required extra wiring and the extensions are very difficult to manage. The solution to this problem is the use of Internet protocol which carries voice as a data called "Voice over Internet Protocol". The advantage of this system is that it uses the existing network connection installed for the organization and the management of the extensions are very easy as it can be manages from the web browser. The system is cost efficient as it uses only one computer system as a server with Linux based operating system called "trixbox". The system uses the USB handset as an interfacing device like telephone.
\end{abstract}

\section{General Terms}

Voice over Internet Protocol and Session Initiation Protocol i.e. SIP based client server system architecture is the base of the system. The System is compatible for LAN, MAN and WAN. This system also supports the Voice over WiFi Network if the organization consists of WiFi campus.

\section{Keywords}

SIP, VoIP, trixbox, USB handset.

\section{INTRODUCTION}

Voice Over Internet Protocol deals with the conversion of analog audio signals into the digital data that can be transmitted over the internet by using internet protocol. VoIP turns the standard internet connection into the phone calls [2]. Many organizations uses Electronic Private Branch Exchange System for the communication using extension numbers assigned to the users. It utilizes the man power and extra wiring for the installation as well as it doesn't support the advance facilities like call waiting, voicemail, caller ID etc. Its main disadvantage is that the change of extension is very difficult task. The Private Branch Exchange run on VoIP telephony provides the organization the sophisticated installation and configuration of the user extensions [2]. This technology reduces the cost and the time of the installation and configuration, it doesn't require that much manpower as EPBX system. The project aim is the implementation of the VoIP telephony system software and its configuration. The base is the operating system called "trixbox" which is Linux based VoIP PBX server operating system. These operating systems consist of the telephony package called "Asterisk". This package consists of several features such as Voicemail, Call Waiting, Caller ID, Conference, Call Hold, Call Transfer etc. Asterisk supports audio protocols such as SIP which is Session Initiation Protocol used for the audio communication. The VoIP PBX system for the organization use the backbone of Local Area Network on which the extensions were configured using computer system. The "trixbox" server is the Linux based and the clients were the windows based or Linux based using the "Softphone" for the communication. Instead of Softphones the VoIP telephone devices such as USB handset and Hardphones can be used

\section{PBX System}

The typical PBX system consists of an Electronics Switching between the user extensions. In this if we want to add new user the extra switching circuitry must be added and the new wired connection is to provide for the user from the PBX to its location. This is not cost efficient system. Also as per the requirement of the customer the extra features like audio video conferencing, voicemail, Phone book, blacklist and most important easy to manage is not possible with EAPBX system. So the solution is to build such system which provides such features. One of the systems is "trixbox" operating system having all those features including web based server access and management of the extensions which the customer expected.

\section{The "trixbox" System}

The "trixbox" operating system is made up of a number of components each of which is released under an open-source license. In this paper the Trixbox CE V 2.0.8.12 is used. Trixbox is the open source IP telephony software consists of the Asterisk PBX package. The main feature of the operating system is that the sophisticated installation \& configuration of the system with number of advance features like Voicemail, Video conferen cing, Phonebook, Blacklist, Control panel to monitor the extensions, easy to add and delete the user extensions, flexible to move the user extensions without disturbing the existing network.

\subsection{System require ment}

The minimum system required is an Atom processor Board, 160 GB of IDE hard disk, $1 \mathrm{~Gb}$ of RAM, $1 \mathrm{D}$-link Ethernet port, CD ROM, PS/2 Keyboard, Pico PSU 90W, Cabinet and one USB handset. The software required are the "trixbox" Operating system as a server and "X-Lite" softphone as a user extension SIP phone which can be access by USB handset.

\subsection{Installation}

First buy the license copy of the "trixbox" operating system from www.fonality.com we can download the trial version also 
which has a limited features. Burn the ISO image to disk and then assemble the system and power on it. Insert the $\mathrm{CD}$ and the installation begins with the startup screen as shown in figure 1. Just press enter button and the system will be ready in 15 to 25 minutes.

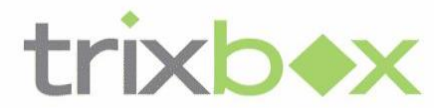

The Open Platform for Business Telephony

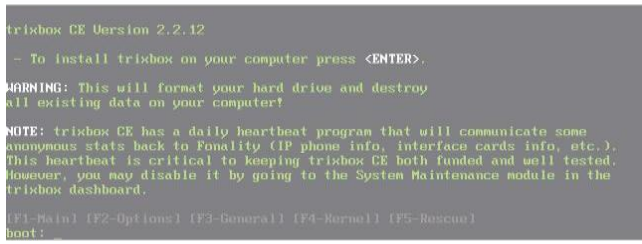

Figure 1 startup screen

While installation configure the TCP/IP address of the system. Give the static IP address which is unique in the network along with the default gateway and primary name server we took an example as shown in the figure 2.

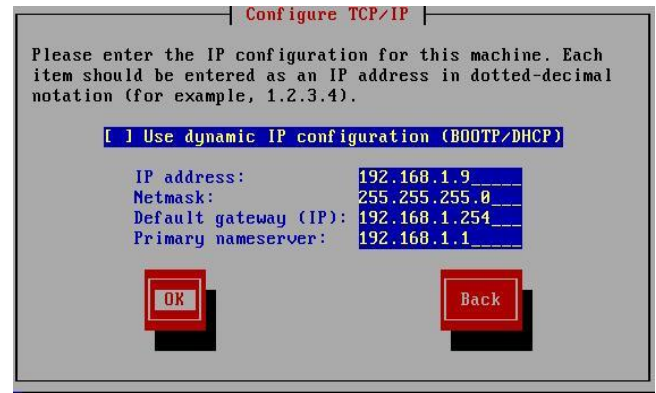

Figure 2 Configuring TCP/IP

\subsection{Accessing the server}

After the installation the server is ready and asks for the login name and password which is given at the time of installation. If the installation is successful then the message - "For access to the trixbox web GUI use this URL http://192.168.1.9" appears. Then go to the client computer which might be any one having the internet browser and it should be of the same network. Type http://192.168.1.9 in the address field it will show the trixbox homepage as shown in figure 3. By clicking "switch" menu it will ask the username and password to log into admin mode. By default the username is "maint" and password is "password" [7]. Then go to Asterisk free PBX option.

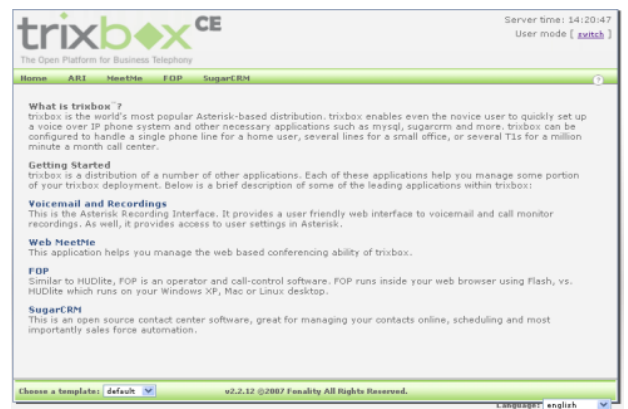

Figure 3 trixbox home page.

\subsection{Extension Configuration}

After switching to "maint" administrator panel go to the setup menu and click on the extensions option. Select the SIP device and click "Add SIP Extension". The screenshot is shown in figure 4 .

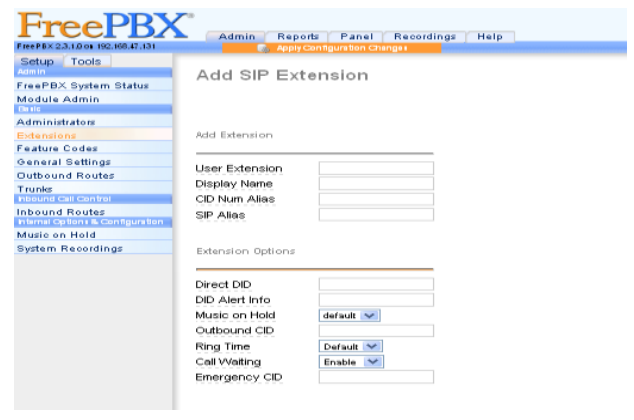

Figure 4 Add SIP Extension

Here add the new extension by giving the user extension say " 100 ", display name say "Alice", secret as the password for the extension. The port address for the system is " 5060 " which is the communication port. Leave other fields blank. We can create more than 10,000 extensions depending on the hardware configuration.

\section{Configuration of SIP Phones}

Now we configure the Softphone on the client/user system which might be windows, Linux or Mac. Buy the software X-lite which is available on www.counterpath.com we can download the trial software having very limited features. Figure 5 shows the X-lite softphone. After the successful installation the extension which is created in server is now configured here by entering the details in SIP proxy setting [3].

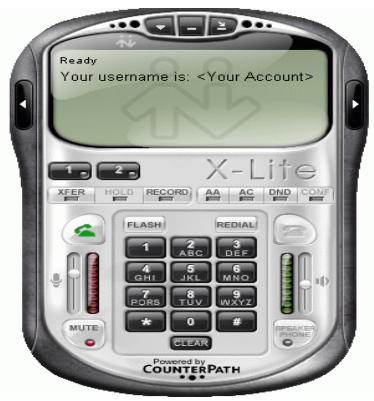

Figure 5 X-Lite Softphone 
Enter the user extension details in the configuration box of $\mathrm{x}$ lite such as -

$\begin{array}{lll}\text { Display name } & : & X Y Z \\ \text { Extension } & : & 100 \\ \text { Secret } & : & * * * * \\ \text { Domain } & : & \text { Default } \\ \text { SIP Proxy } & : & 192.168 .1 .9\end{array}$

After searching the default SIP proxy i.e. trixbox server the user extension is configured. Dial $* 65$ on the phone and you will listen to the user extension number. Similarly configure the other extensions on the different user machine. Now call from the one user to other in the network and the SIP communication is established.

\section{VOICEMAIL}

The one of the important feature of this system is the voicemail. It is beneficial when the user is off line on not responding. The voicemail can be sent to any extension and can be access by the user by using voicemail ID and password. The access to voicemail is permitted by the administrator while configuring the user extension. To give the permission goes to the PBX panel and select the user extension in setup menu or create the new extension. Here go to the field of voicemail and enter the voicemail password by enabling the stat us of voicemail service in the extensions field.[7]

After the configuration the user can access the voicemail service by simply login to the voicemail service in the web browser. The login screen is shown in the figure 6.

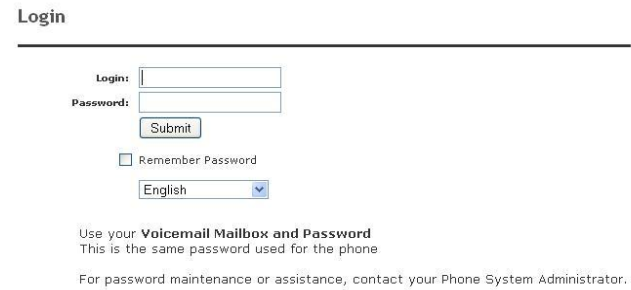

Figure 6 Voicemail login

After entering the user extension say " 100 " for "Alice" and the password provided by the administrator a user could login into its voicemail account to see the voicemail status [7]

\section{CONCLUSION}

This paper describes one solution for a PBX based on existing network infrastructure of an organization. It is a lowcost system and very easy to install and manage.

The system reduces the wiring cost as compare to EAPBX system. The extensions can be easily created, deleted or shifted without disturbing the other communication. The future work would include private branch exchange system based on Wireless Network where the extensions are the wireless pocket device having almost all the features of mobile phone. Mobile phones required the service provider but this system itself the service provider for the created extensions and most important that this system gives the service free of cost for the existing wireless network of an organization.

\section{ACKNOWLEDGMENTS}

We thank our organization for providing the necessary facility for the successful implementation of this system. Our sincere thanks to the "trixbox" team and "counterpath" team for providing the guideline for the installation of the system.

\section{REFERENCES}

[1] Md. Zaidul Alam, Sau gata Bose, Md. Mhafuzur Rahman, Mohammad Abdullah Al-Mumin "Small Office PBX Using Voice Over Internet Protocol" in ICACT 2007.

[2] BUR GOODE, SENIOR MEMBER, IEEE "Voice Over Internet Protocol (VoIP)" in PROCEEDINGS OF THE IEEE, VOL. 90, NO. 9, SEPTEMBER 2002

[3] J. Rosenberg, H. Schulzrinne, Camarillo, Johnston, Peterson Sparks, Handley, and Schooler, "SIP: Session initiation protocol v.2.0," IETF RFC 3261, 2002

[4] “A CRM model based on Voice over IP". Y.S. Moon, C.C. Leung, K.N. Yuen', H.C. Ho, X. Yu Department of Computer Science and Engineering, Dept. of System Engin. and Engin. Management, The Chinese University of Hong Kong Shatin, N.T., Hong Kong. IEEE 2000

[5] "SMALL OFFICE PBX USING VOICE OVER INTERNET PROTOCOL (VOIP)" Md. Zaidul Alam, Saugata Bose, Md. Mhafuzur Rahman, Mohammad Abdullah Al-Mumin ICACT 2007

[6] "Conferencing, Paging, Voice Mailing via Asterisk EPBX" Ale Imran1, Mohammed A Qadeer2, 2009 International Conference on Computer Engineering and Technology

[7] "trixbox made easy" Barrie Dempster and Kerry Garrison , Packet Publication 2006

[8] Analysis of Telephone System of a University Campus and Design of a Converged VoIP System Amel Chowdhury (Corresponding author), Jakia Afruz ,Jalalur Rahman Department of Applied Physics, Electronics and Communication Engineering University of Dhaka, Dhaka1000, Bangladesh Computer \& Information Science Jpurnal Vol 1 No.4, Nov 2008.

[9] Design and Implementation of an Embedded VoIP System using Bluetooth Technique YU Jijiang Computer and Information Engineering Department \& LIAN Fiyu College of Information Science and Technology Henan University of Technology Zhengzhou, China 2010 2nd International Conference on Future Computer and Communication V3-345.

[10] ASR System Integration with Asterisk for SIP or IAX Softphone clients, 2009 International Association of Computer Science and Information Technology - Spring Conference Saurabh Goel, Vikash Garg, Prashant Ranjan, Satyanaray an Rao, Mahua Bhattacharya, Department of Information Technology, Indian Institute of Information Technology and Management, Gwalior, MP, INDIA 